\title{
Two Clonal Species of Phytophthora Associated to Solanaceous Crops Coexist in Central and Southern Colombia
}

\author{
Sandra Catalina Chaves, ${ }^{1}$ Natalia Guayazán, ${ }^{1}$ Maria Fernanda Mideros, ${ }^{1}$ Mayra Parra, ${ }^{1}$ \\ Florencia Lucca, ${ }^{2}$ and Silvia Restrepo ${ }^{1, \dagger}$ \\ ${ }^{1}$ Departmento de Ingeniería Química, Universidad de los Andes, Bogotá, Colombia \\ ${ }^{2}$ Instituto Nacional de Tecnología Agropecuaria, Estación Experimental Agropecuaria Balcarce, República, Argentina \\ Accepted for publication 25 March 2020.
}

\begin{abstract}
In Colombia, late blight is considered one of the most limiting diseases on potato and tomato production. Recently, a new Phytophthora species, $P$. betacei, was described infecting tree tomato crops in the south of Colombia. However, the distribution and the host range of this new emerging pathogen in the country are unknown. The main aims of this study were to determine if this novel species is confined to the south of Colombia, to assess if $P$. betacei represents a genetically uniform clone across Colombia and to determine if in all regions there is a clear differentiation between the two Phytophthora species. Therefore, we characterized Phytophthora isolates obtained from tree tomato and potato

simple sequence repeat markers. Results showed a strong population structure between $P$. infestans and $P$. betacei. However, we did not detect any genetic differentiation within $P$. infestans or $P$. betacei populations from different regions. Furthermore, we detected significant morphological differences among the species based on growth and sporangial morphology measurements. We also showed that strains of Phytophthora spp. are predominantly of the A1 mating type and belong to EC- 1 and EC-3 clonal lineages for $P$. infestans and P. betacei, respectively. Our results describe the expanded geographical range of the new species of $P$. betacei in the central region of Colombia.
\end{abstract} crops in a central region of Colombia and compared them with the strains from the south. Initially, we evaluated the genetic differentiation among Phytophthora strains obtained from tree tomato and potato crops using
Keywords: ecology and epidemiology, etiology, geographical range, Phytophthora betacei, population biology, population structure
The genus Phytophthora (from the Greek for "plant destroyer") comprises species that are the most important plant pathogens worldwide (Fry 2008). Within the genus there are 150 formally described species, including the pathogens $P$. infestans, $P$. sojae, $P$. ramorum, and $P$. cinnamomi (Cavalier-Smith 1993; Dick 1995; Yoon et al. 2002). Among these, P. infestans is the most widely known plant pathogen (Fry 2008) and it is characterized by the ability to cause disease in several hosts within the family Solanaceae, including potato (Solanum tuberosum), yellow potato (Solanum phureja), and tomato (Solanum lycopersicum) (Adler et al. 2004). This pathogen is responsible for causing large economic losses in potato and tomato crops and has been considered a major threat to global food security ever since the Irish potato famine of the mid-19th century (Fry 2008).

In an effort to classify Phytophthora species, previous studies grouped them into eight main clades based on sequence analyses of the internal transcribed spacer regions (ITS1 and ITS2) in the rRNA genes (Kroon et al. 2004). Furthermore, based on cytochrome oxidase I and II genes (coxI and coxII) (Martin et al. 2004) the first group was further divided into three subclades: $1 \mathrm{a}, 1 \mathrm{~b}$, and $1 \mathrm{c}$. $P$. infestans has been located in the 1c clade together with $P$. mirabilis, $P$. phaseoli, $P$. ipomoeae, as well as the recently described species

${ }^{\dagger}$ Corresponding author: S. Restrepo; srestrep@uniandes.edu.co

Funding: This work was supported by the Department of Biological Sciences at Universidad de los Andes. Additional funding for this research was provided by the Research Fund of the School of Sciences and the Office of the Vice President for Research from Universidad de los Andes. Colombian Phytophthora betacei strains are included in "Contratos de Acceso a Recursos Genéticos y sus productos derivados", $\mathrm{N}^{\circ} 128,23 / 09 / 2016-\mathrm{MADS}-\mathrm{SRR}$ and $\mathrm{N}^{\circ} 211,11 / 07 / 2018$.

*The $e$-Xtra logo stands for "electronic extra" and indicates that supplementary materials are published online.

The author(s) declare no conflict of interest.

(c) 2020 The American Phytopathological Society
$P$. andina and P. betacei (Gómez-Alpizar et al. 2008; Kroon et al. 2004; Martin et al. 2004; Mideros et al. 2018; Oliva et al. 2010).

In South America, $P$. infestans, $P$. andina, and $P$. betace $i$ from the $1 \mathrm{c}$ clade have been reported attacking plants within the family Solanaceae. In the case of $P$. infestans, several studies have described the highly clonal population structure of this pathogen in Peru, Ecuador, Chile, Brazil, Venezuela, and Bolivia (Cárdenas et al. 2011; Forbes et al. 1997; Santana and Gomes 2013). In Ecuador, the EC-1 clonal lineage commonly associated with potato and yellow potato, and the US-1 clonal lineage associated with tomato and sweet cucumber (Solanum muricatum) have been reported (Oyarzún et al. 1998). Similarly, the Colombian populations of $P$. infestans on potatoes are represented by the EC-1 clonal lineage (Vargas et al. 2009). In Ecuador, Peru, and Colombia, in addition to $P$. infestans, $P$. andina was reported infecting tree tomato, sweet cucumber, lulo, or naranjillo (Solanum quitoense), and wild plants belonging to the genus Anarrhichomenum (GómezAlpizar et al. 2008). $P$. andina was initially reported as a polyphyletic species and represented two clonal lineages, the EC2 clonal lineage for strains that infect wild solanaceous species and the EC-3 clonal linage reported in strains infecting tree tomato crops (Adler et al. 2004; Forbes et al. 1997; Ordoñez et al. 2000; Silva et al. 2009). Then, P. betacei was reported in South America attacking tree tomato crops. This species is closely related to $P$. infestans and $P$. andina and is characterized by the EC- 3 clonal lineage (Mideros et al. 2018). This study suggested that the initial description of $P$. andina included strains from the EC-3 clonal lineage, which should instead be classified as $P$. betacei (Mideros et al.2018). $P$. betace $i$ is heterothallic with a low oospore production and an abnormal appearance when crossed with a $P$. infestans strain of the A2 mating type (Mideros et al. 2018).

$P$. betace $i$ has only been reported in the south of Colombia, where it is restricted to tree tomato crops (Mideros et al. 2018) and no further studies have been performed since 2018. Therefore, we aimed to assess if this novel species is confined to the south of 
Colombia or whether it could be found associated with other crops in other regions of Colombia. Also, we aimed to know if $P$. betacei represents a genetically uniform clone across Colombia and whether it also infects other hosts. Finally, we assessed the differentiation between potato and tree tomato strains in different regions of Colombia.

\section{MATERIALS AND METHODS}

Collection of Phytophthora strains. A collection of 157 Phytophthora strains from $P$. infestans, $P$. betacei, and $P$. andina was included in this study. Each strain belonged to one of the following regions: central Colombia (Cundinamarca State), southern Colombia (Nariño and Putumayo states) (Fig. 1), the United States (P. infestans), and Ecuador (P. andina) (Supplementary Table S1).

A total of 98 isolates of $P$. infestans and $P$. betacei from the central and southern regions were collected in this study between May 2012 and October 2016 (Supplementary Table S1). For this, potato (both species, $S$. tuberosum and $S$. phureja) and tree tomato crops were sampled, and three to four leaves from 15 randomly selected plants showing late blight symptoms were collected (Supplementary Table S1). To obtain $P$. infestans strains, three pieces $\left(1 \mathrm{~cm}^{2}\right.$ each) were excised from each infected leaf between the necrotic and sporulating area and placed on potato dextrose agar medium (PDA) amended with antibiotics (rifampicin at $0.0125 \mathrm{~g} /$ liter, ampicillin at $0.1 \mathrm{~g} / \mathrm{liter}$, chloramphenicol at $0.25 \mathrm{~g} /$ liter, and pentachloronitrobenzene at $0.0125 \mathrm{~g} /$ liter) (Danies et al. 2013). Once growth was observed, monocultures were obtained by transferring one single sporangium onto a fresh PDA medium. P. betacei strains were obtained similarly using tree tomato agar (TTA) medium amended with the same antibiotics. All the plates were grown at $18^{\circ} \mathrm{C}$ in the dark for 7 days. All the new strains were stored in the Museum of Natural History at Universidad de los Andes under the accession codes ANDES F1081 to ANDES F1132 and ANDES F1133 to ANDESF1195.

All $P$. betacei from the southern region as well as $P$. andina from Ecuador and $P$. infestans from the United States region were obtained from the Phytophthora collection stored in the Museum of Natural History at Universidad de los Andes (MNHUA) (Mideros et al. 2018) or provided by William Fry, Cornell University (Supplementary Table S1).

Molecular characterization and population genetics analyses. DNA extraction. Agar plugs of each Phytophthora spp. strain, taken from the edge of a 7-day-old actively growing colony on PDA or TTA, were transferred to plich broth $(12.5 \mathrm{~g}$ liter $^{-1}$ of saccharose, $0.25 \mathrm{~g} \mathrm{liter}^{-1}$ of yeast extract, $5 \mathrm{mg}$ liter $^{-1}$ of $\beta$-sitosterol, $0.5 \mathrm{~g} \mathrm{liter}^{-1}$ of L-asparagine, and $5 \mathrm{ml} \mathrm{liter}^{-1}$ of thymine). After 20 days of incubation, all the mycelia were harvested and lyophilized, and DNA was then extracted using the DNeasy Plant Mini kit (Qiagen, Germantown, MD, U.S.A.) following the manufacturer's instructions. The DNA was suspended in Tris-EDTA buffer ( $\mathrm{pH}$ 8.0) and treated with RNase. DNA quality and quantity were assessed using a NanoDrop ND-1000 and visualized in $1 \%$ agarose electrophoretic gel. Treated DNA samples were stored at $-20^{\circ} \mathrm{C}$ until needed.

RG57 clonal lineage determination. The clonal lineage for the Phytophthora strains was determined via a restriction fragment length polymorphism (RFLP) analysis using the RG57 probe (Goodwin et al. 1992). Southern blot analysis was conducted using the Amersham gene images AlkPhos direct labeling and detection system (GE Healthcare) according to the manufacturer's instructions. The Netherlands strain NL13316, US-17 genotype (US970001); the US-8 genotype (US400994); and the US-1 genotype (SA960008) were used as reference strains in RG57 analyses. The presence or absence of known fingerprint fragments was scored visually.

Mitochondrial haplotype determination. The mitochondrial haplotype of each strain was determined following the protocol described by Griffith and Shaw (1998). This protocol was designed to determine the mitochondrial DNA haplotype of $P$. infestans strains using a polymerase chain reaction (PCR), followed by RFLP analysis using four primer pairs (F1-R1 CfoI, F2-R2 MspI, F3-R3 EcoRI, and F4-R4 EcoRI). Reference strains US970001 (US-17) and US040009 (US-8) were included in this analysis as controls for mitochondrial haplotype Ia (Danies et al. 2014).

Simple sequence repeat (SSR) amplification. All strains were genotyped using a set of 12 polymorphic SSR markers (Cooke et al. 2012; Goss et al. 2014; Knapova and Gisi 2002; Lees et al. 2006; Li et al. 2013). The microsatellite markers used were D13, G11, Pi04, Pi4B, Pi63, Pi70, PinfSSR2, PinfSSR3-Pi02, PinfSSR4, PinfSSR6, PinfSSR8, and PinfSSR11 (Li et al. 2013) (Supplementary Table S2). PCR conditions were as follows: $95^{\circ} \mathrm{C}$ for $5 \mathrm{~min}$ followed by 30 cycles of $95^{\circ} \mathrm{C}$ for $30 \mathrm{~s}, 58^{\circ} \mathrm{C}$ for $90 \mathrm{~s}, 72^{\circ} \mathrm{C}$ for $20 \mathrm{~s}$, and a final extension at $60^{\circ} \mathrm{C}$ for $30 \mathrm{~min}$. PCR products were analyzed on an ABI 3730xl capillary system with POP-7 Polymer (ABI, PN 4335615). PCR amplicons were compared with a set of size standards and alleles were scored accordingly (Danies et al. 2014; Li et al. 2013). At least one reference strain from $P$. infestans of the US- 8 (strain US100028) and US-17 (strain US970001) clonal lineages was included in all microsatellite analyses (Danies et al. 2014), as well as data from P. andina previously reported (Goss et al. 2011; Oliva et al. 2010). Alleles were scored by assigning specific allele sizes using GeneMapper v4.0 software (Applied Biosystems).

Genetic data analysis. Population genetics and structure were assessed using the poppr package version 2.5.0 (Kamvar et al. 2014) executed in R v3.5.1 (R Core Team 2013). For each strain, one specific multilocus genotype (MLG) was assigned according to the default parameters.

To infer between-population relationships, microsatellite-based genetic distances were computed using Bruvo's distance (Bruvo et al. 2004). To eliminate the bias imposed by the presence of clones, a dataset was constructed using the poppr function 'clonecorrect()' including only one representative strain of each MLG according to species, geographic origin, and host. (Grünwald and Hoheisel 2006; Kumar et al. 1999). To visualize genetic distance among MLGs, a minimum-spanning network was created via igraph (Csardi and Nepusz 2006) in poppr (Kamvar et al. 2014).

Genotypic diversity measures were computed for all the strains according to species, geographic origin and host. Nei's unbiased gene diversity (Hexp) (Nei 1987), the Shannon-Wiener index of MLG diversity (H) (Shannon 2001), and evenness (E.5) were calculated with and without correction for sample size (Chao and Shen 2003). Indeed, an eMLG was calculated, and it corresponds to the number of expected MLG at the smallest sample size $\geq 10$ based on rarefaction. Additionally, the Stoddart and Taylor's index of MLG, diversity (G) (Stoddart and Taylor 1988) was calculated to compare the ratio of observed multilocus genotypic diversity to that expected under conditions of sexual reproduction.

Clonality was estimated based on the standardized index of association ${ }_{d}$ (Agapow and Burt 2001) according to species, geographic origin and host. $P$ values were estimated using a onesided permutation test based on 999 samples.

To visually assess between-population differentiations and to determine the contribution of individual alleles to population structure, we conducted two independent principal component analyses (PCA), using the R package adegenet (Jombart 2008), and plotted all individuals in the most significant axes space according to their genetic similarities. Initially, we evaluated the differentiation among strains collected in Colombia from different hosts (potato and tree tomato crops) and geographic origin (central and southern region). Then, we evaluated the differentiation between the strains collected in Colombia, and all Phytophthora species reported in South America: $P$. infestans, $P$. betacei, and $P$. andina strains from tree tomato and the Anarrhichomenum complex and three strains collected in the United States from S. lycopersicum. The Anarrhichomenum complex comprises 10 to 20 viny species found in mountain areas from Mexico to Bolivia (Correll 1962) and several $P$. andina isolates have been collected from these plant species. 


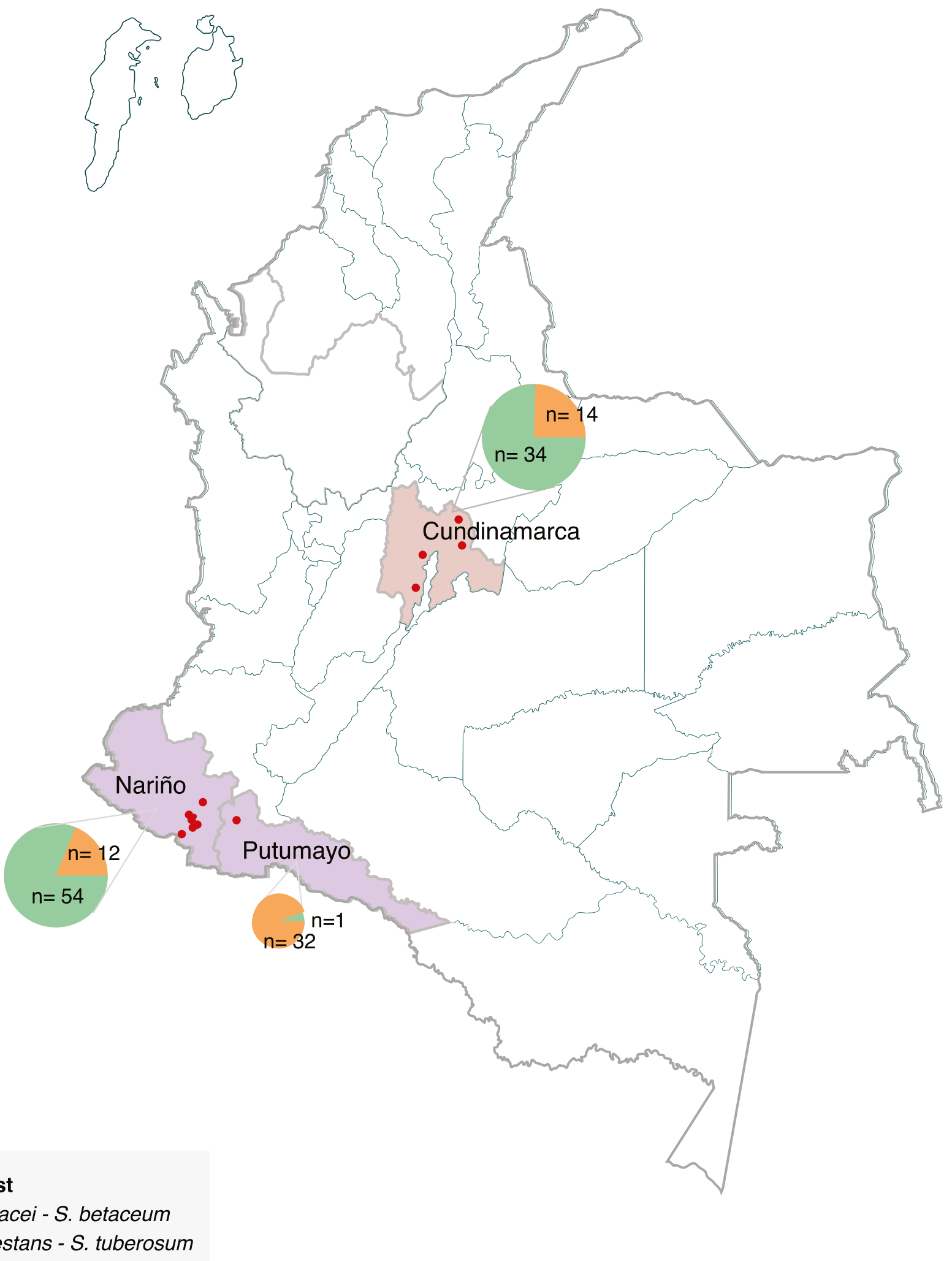

\author{
Isolate - Host \\ $P$. betacei - S. betaceum \\ $P$. infestans - S. tuberosum
}

\title{
Region
}

Central Region - CR

South Region - SR

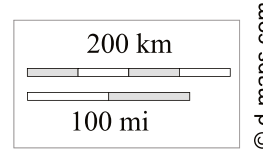

- Sampling site

Fig. 1. Geographic distribution of sampling sites. Phytophthora betacei and P. infestans were collected in different regions of Colombia. In this study, we collected samples in the departments of Cundinamarca and included samples collected from Nariño in a previous study (Mideros et al. 2018). 
Morphological characterization of the strains. Mycelial radial growth and sporangia morphology. To detect differences on mycelial radial growth and sporangial morphology between Phytophthora strains obtained from potato and tree tomato crops from all the geographical regions, strains were grown on PDA, TTA, and corn meal agar (CMA) media (Mideros et al. 2018) as follows: a mycelial plug of $5 \mathrm{~mm}$ in diameter of each strain was transferred to petri plates containing one of the three culturing media and incubated at $18^{\circ} \mathrm{C}$ in complete darkness for 15 days. Mycelial radial growth for each strain-medium combination was registered 15 days after incubation by taking photographs using a Canon Digital EOS Rebel T3i/600D camera (Tokyo, Japan), and then calculated by measuring the total mycelial growth area $\left(\mathrm{cm}^{2}\right)$ using the software ImageJ (Rueden et al. 2017; Schindelin et al. 2015). For sporangia morphology, differences in length $(\mu \mathrm{m})$, width $(\mu \mathrm{m})$, length/width ratio, and area $\left(\mu \mathrm{m}^{2}\right)$ were determined for each strain-medium combination. Mycelia from 15day-old actively growing colony margins were excised and immersed directly in $\sim 1 \mathrm{ml}$ of sterile distilled water. Between 10 to 30 sporangia were measured for each strain-medium combination. Each sporangium was photographed using an optical microscope at $40 \times$, Olympus IX71, and measurements for each variable were taken using ImageJ (Rueden et al. 2017; Schindelin et al. 2015). Each strain was evaluated with two independent replicates on each medium (Mideros et al. 2018; Montarry et al. 2007).

Mating type determination. Mating type of all Phytophthora strains collected from potato and tomato was determined by pairing an unknown strain with a known strain of $P$. infestans, either A1 mating type (US970001 US-17 genotype) or A2 mating type (US040009, US-8 genotype). In vitro crosses were performed on 1/ 2-V8-rye medium (50\% clarified rye, $50 \%$ clarified V8, $1.5 \%$ agar, and $1 \mathrm{ml}$ of $\beta$-sitosterol per liter, $\mathrm{pH}$ 8.5) (Mayton et al. 2000; Mideros et al. 2018). Petri dishes were kept at $18.5^{\circ} \mathrm{C}$ for 15 to 30 days. The hyphal interface of the two colonies was investigated microscopically using $125 \times$ magnification. Strains that formed oospores at the interface with the known A1 strain were designated A2, and those that formed oospores with the known A2 strain were designated A1. The known strains (A1 and A2) were paired as positive controls, while negative controls consisted of pairing the known strains with themselves (same mating type) (Danies et al. 2014; Mayton et al. 2000).

Statistical analyses of the morphological data. All statistical analyses were conducted using the software R, version 3.5.1 (R Core Team 2013), and the significance threshold was fixed at $\alpha=$ 0.01 . To quantify the variation in colony and sporangia morphology, we fitted two full factorial linear mixed models using the 'lmer' function in the R package 'Ime4' (Bates et al. 2014). In the models, either mycelial radial growth or sporangia morphology measurements were the response variables, species, geographical region, media, and their interactions were considered as fixed effects while the strain was considered random and was nested within the species.

$$
\text { trait } \sim \operatorname{species}_{i}+\text { region }_{j}+\text { media }_{k}+(\text { species } \times \text { region })_{i j}
$$$$
+(\text { species } \times \text { region } \times \text { media })_{i j k}+\operatorname{species}(\operatorname{strain})_{l}+\varepsilon_{i j k l}
$$

Normality of the residuals of all the linear models was determined. In all cases, they were not normally distributed (Shapiro-Wilk test; $P<0.01$; Supplementary Table S3) and thus we used the nonparametric rank sum tests Kruskal-Wallis implemented in the $\mathrm{R}$ package 'stats' (R Core Team 2013) to compare the calculations of colony growth and sporangia morphology on each media among species and regions. To detect pairwise differences, we performed multiple comparisons using nonparametric Nemenyi post hoc tests for Kruskal-Wallis and corrected the $P$ values using the Bonferroni method in the 'PMCMRplus' package implemented in R (Pohlert 2014).
Lastly, we established whether the morphology of $P$. betace $i$ and other Phytophthora species differed by visualizing all the sporangial morphology traits in a bidimensional plane. We used a linear discriminant function analysis (LDA) based on the linear combination of morphological variables to assess whether morphological traits separated $P$. betacei from $P$. andina and $P$. infestans, and populations from different geographical regions: central and southern Colombia, Ecuador, and the United States. To this end, we produced a matrix with four traits (sporangia length, sporangia width, sporangia area, and the sporangia length/width ratio) and a total of 27 individuals (nine strains for $P$. betacei, 15 strains for $P$. infestans, and three strains for $P$. andina). Analyses were conducted using the "lda" function from the package 'mass' in R (Venables and Ripley 2002).

\section{RESULTS}

Strain collection. In this study, a total of $14 P$. betacei and 34 $P$. infestans strains were collected in the central region (Cundinamarca State) and $55 P$. infestans strains were collected in the south of Colombia. Furthermore, 44 P. betacei strains from southern Colombia (Putumayo and Nariño States) were included in this study (Mideros et al. 2018) (Fig. 1; Supplementary Table S1). A total of 147 Colombian strains (Fig. 1), three from the United States, and seven from Ecuador were investigated in this study (Supplementary Table S1).

All strains collected from potato in this study belonged to the RG57 clonal lineage EC-1 and the mitochondrial haplotype IIa, while the strains collected from tree tomato plants belonged to RG57 clonal lineage EC-3 and mitochondrial haplotype Ia. Mating types are reported in Supplementary Table S1. All strains collected in this study belong to the A1 mating type.

Population genetic analyses. Twelve microsatellite loci were used to genotype 157 Phytophthora spp. strains included in this study. Microsatellite loci were polymorphic for all individuals genotyped. The number of alleles per locus ranged from two to nine with a mean value of 4.25 . Loci D13, SSR4, and G11 were the most informative with 11, nine and eight alleles, respectively, whereas loci Pi70 and Pi04 were the least informative with two alleles. Furthermore, loci D13, Pi63, Pi70, PiG11, SSR11, SSR2, SSR6, SSR8, and SSR3-Pi02 showed to be diploid; meanwhile loci Pi4B and SSR4 showed evidence of triploidy.

In total, 64 MLG were detected among all individuals for all the microsatellites examined. A total of 56 MLGs were detected among all the strains collected from both the central and southern regions of Colombia, 18 MLG among all individuals collected only from the central region, and 38 MLG from the southern region. Furthermore, the number of MLGs detected among strains collected from potato and tree tomato was 46 and 10, respectively (Table 1).

The SSR markers used in this study showed the genetic diversity within Phytophthora species in Colombia. Strains collected from potato exhibited higher diversity than strains collected from tree tomato (Table 1). Values of $\mathrm{H}$ varied from 2.861 to 2,425 for strains collected on potato and from 1.509 to 0.656 for strains collected on tree tomato from the southern and central region, respectively. Genetic diversity for Phytophthora spp. strains calculated according to Hexp, ranged from 0.382 to 0.387 on potato and from 0.278 to 0.292 on tree tomato from the southern and central region, respectively (Table 1 ).

When we compared genotypic diversity between $P$. betacei, $P$. infestans, and $P$. andina, we found the highest value for $\mathrm{G}$ in strains identified as $P$. infestans (9.03), indicating that this is the most diverse population. On the other hand, the population of $P$. andina showed an intermediate value (5.44). P. betacei showed low diversity for all the indexes (Table 1).

A lack of sexual reproduction was detected based on the results for the linkage analysis among the markers, for almost all regions. Clone-corrected and uncorrected data rejected the hypothesis of no 
linkage among markers except for $P$. infestans in southern Colombia (Supplementary Table S4). We thus have support to describe a single large population of $P$. infestans on potato and a population of $P$. betace $i$ on tree tomato. $P$. infestans clonality measured with ${ }_{d}$ varied from 0.1878 to 0.0682 (clone-corrected) in central and southern regions, respectively. On the other hand, P. betacei showed values of ${ }_{d}$ ranging from 0.7805 to -0.0281 in central and southern regions, respectively. Multilocus linkage disequilibrium was significantly greater than zero within each population according to the geographic location $(P<0.001)$.

We also detected variation in the genetic diversity according to the geographical origin of the strains from Colombia. On potato crops, values of $\mathrm{G}$ evaluated were higher for strains collected from the central region than strains collected in the southern region of Colombia (Table 1), while values of $\mathrm{H}$ showed the opposite. On the other hand, for strains collected on tree tomato, higher genetic diversity was detected in the southern region compared with the central region (Table 1).
Genetic differentiation. The PCA conducted using microsatellite data, clearly differentiated strains collected from potato (S. tuberosum) and tree tomato crops in the central and southern regions of Colombia. The results showed two genetic clusters corresponding to different species: $P$. infestans (associated with potato) and $P$. betacei (associated with tree tomato). PC1 separated $P$. infestans and $P$. betace $i$ (mean variance explained $=50.1 \%$ ), indicating that a large proportion of the genetic variation is explained by the genetic differentiation between strains belonging to these two species. The PC2 (mean variance explained $=5.4 \%$ ) showed geographic variation within $P$. infestans and $P$. betacei according to central (upper) and southern (lower) regions (Fig. 2A).

Furthermore, we confirmed the differentiation between the strains collected from Colombia, and other Phytophthora species reported in South America and strains from the United States. Results obtained from the PCA (Fig. 2B) showed that P. infestans, $P$. betacei, and $P$. andina are completely distinct genetic units. The PC1 separated $P$. betace $i$ and $P$. infestans (mean variance explained

TABLE 1. Genotypic diversity statistics for all Phytophthora species reported in South America in P. infestans, P. betacei, and P. andina and three strains collected in the United States ${ }^{\mathrm{a}}$

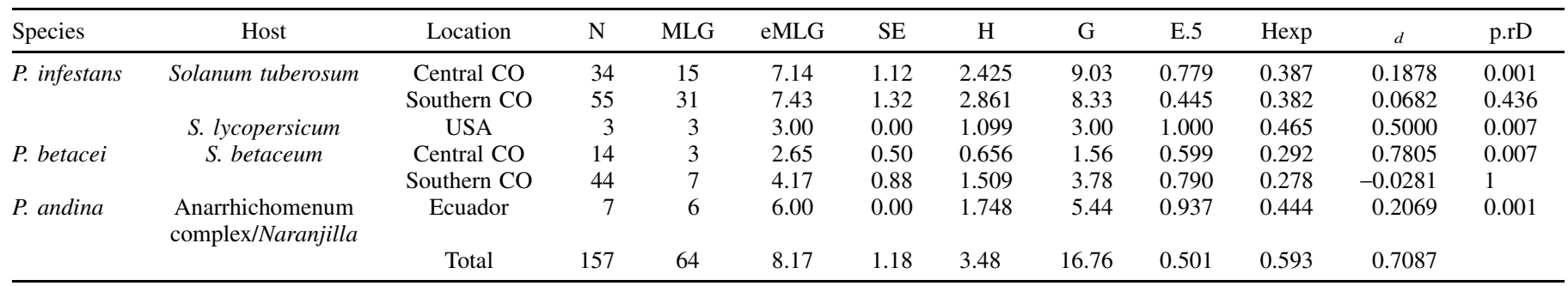

${ }^{a}$ Results were obtained from the simple sequence repeat analysis using poppr package (Kamvar et al. 2014). Clone dataset corrected. $\mathrm{N}=$ number of individuals observed, MLG = number of multilocus genotypes (MLG) observed, eMLG = number of expected MLG at the smallest sample size $\geq 10$ based on rarefaction, SE = standard error based on eMLG, $\mathrm{H}=$ Shannon-Wiener index of MLG diversity, G = Stoddart and Taylor's index of MLG diversity, E.5 = evenness $E_{5}$, Hexp = Nei's unbiased gene diversity, rbarD $=$ the standardized index of association ${ }_{d}$, p.rD $=P$ value for ${ }_{d}$, and $\mathrm{CO}=\mathrm{Colombia}$.

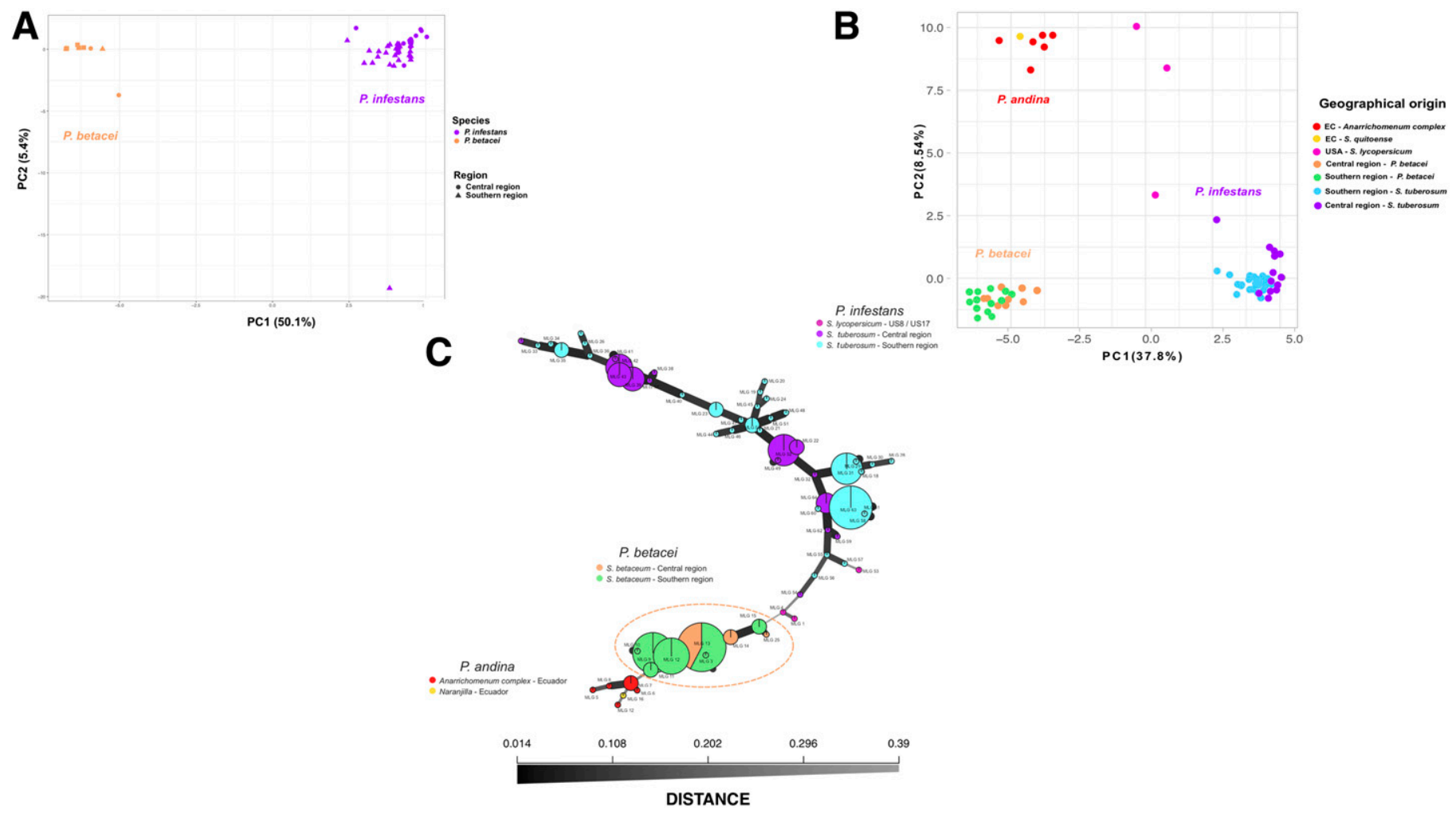

Fig. 2. A, Principal component analysis (PCA) discriminating Colombian strains collected from central and southern regions, B, PCA discriminating all Phytophthora species reported in South America (P. betacei, P. infestans, and P. andina and three strains collected in the United States), and C, minimum spanning network using Bruvo's distance. 
$=37.8 \%$ ), indicating that the main genetic variation is explained by differences between strains belonging to these two species. PC2 (mean variance explained $=8.54 \%$ ) separated $P$. andina strains collected from $S$. quitoense and Anarrhichomenum complex from $P$. betacei and $P$. infestans. The PCA results showed that the observed genetic differentiation is best explained by a separation among $P$. infestans, $P$. andina, and $P$. betacei (Fig. 2B). The results observed in the minimum-spanning network further supported that $P$. infestans, $P$. betacei, and $P$. andina are discrete genetic units as clearly depicted by three separate nodes (Fig. 2C).

Morphological characterization of the Phytophthora spp. strains. With the aim to evaluate whether there were significant differences among $P$. betacei, $P$. infestans, and $P$. andina from four geographical regions, we evaluated five morphological traits. Results from the Kruskal-Wallis rank sum test followed by pairwise comparisons using the Nemenyi test indicated that the species (Table 2) and the regions (Table 3 ) influenced the mycelial radial growth of the Phytophthora species on PDA, CMA, and TTA (Fig. $3)$. Individual comparisons between species revealed that mycelial radial growth of $P$. infestans on PDA was significantly larger than that of $P$. betacei $(P<0.01)$; on CMA, $P$. andina was also significantly larger than $P$. infestans and $P$. betacei $(P<0.01)$. No significant differences were found on TTA. Furthermore, mycelial radial growth on PDA was significantly larger $(P<0.01)$ on $P$. betacei strains collected from the central region compared with the south; on CMA, the pattern was opposite. Similarly, significant differences were found in sporangial morphology among species-media combinations (Table 4), where $P$. betacei and $P$. andina showed to have the largest sporangia in terms of length, length/width ratio, and area, while $P$. infestans displayed the smallest sporangia among the three species. Interestingly, the media had a more dramatic effect on the sporangia morphology of $P$. betacei compared with the other species, where the size was considerably decreased in sporangia harvested from individuals grown on PDA. Furthermore, significant differences were found within species in all the region-media combinations and comparisons for all the sporangial morphology traits (Supplementary Table S5), except for $P$. betacei from the central and southern region, as no sporangia were observed on PDA. Other sporangia morphology differences detected within $P$. betace $i$ strains from different regions were small but significant $(P<0.01)$.

A multivariate LDA, was used to detect morphological differences among Phytophthora strains by maximizing the separation between species based on their sporangial morphology. All sporangial morphology traits were included in the analysis (length, width, length/width ratio, and area of sporangia). The results of the LDA showed that the first and second components accounted for $94.21 \%$ (LD1) and $5.79 \%$ (LD2) of the total variation, respectively. Based on the coefficients of the linear discriminants, length/width ratio of sporangia was the most informative trait for differentiation of the species. Meanwhile, length, width and area contributed only slightly to the species differentiation (Table 5). P. betacei and P. infestans could be differentiated into two discrete groups when all morphological traits were analyzed jointly. However, this analysis did not place $P$. andina strains in a separate cluster. Conversely, there was no evident morphological separation of strains based on their geographical origin (Fig. 4).

In vitro mating type analyses showed that all Phytophthora strains collected from Colombia in this study belonged to the A1 mating type (Supplementary Table S1). Mating type for all other strains had been previously reported elsewhere (Goss et al. 2011; Mideros et al. 2018; Oliva et al. 2010; Vargas et al. 2009).

\section{DISCUSSION}

In this study we characterized strains of Phytophthora spp. associated with potato and tree tomato crops in Colombia using both

TABLE 2. Mycelial radial growth differs among Phytophthora betacei, $P$. andina, and P. infestans strains ${ }^{\mathrm{a}}$

\begin{tabular}{|c|c|c|c|c|c|c|c|c|}
\hline \multirow[b]{3}{*}{ Medium } & & & & \multicolumn{2}{|c|}{$\begin{array}{c}\text { Kruskal-Wallis rank } \\
\text { sum test }\end{array}$} & \multicolumn{3}{|c|}{ Pairwise comparisons using Nemenyi test } \\
\hline & \multicolumn{3}{|c|}{ Median radial growth $\left(\mathrm{cm}^{2}\right)$} & \multirow{2}{*}{$\begin{array}{c}\chi^{2}, \\
\mathrm{df}=2\end{array}$} & \multirow[b]{2}{*}{$P$ value } & \multirow{2}{*}{$\begin{array}{l}\text { P. betacei versus } \\
\text { P. andina }\end{array}$} & \multirow{2}{*}{$\begin{array}{c}\text { P. infestans versus } \\
\text { P. andina }\end{array}$} & \multirow{2}{*}{$\begin{array}{c}\text { P. infestans versus } \\
\text { P. betacei }\end{array}$} \\
\hline & P. betacei & P. infestans & P. andina & & & & & \\
\hline PDA & $2.05( \pm 23.02)$ & $31.93( \pm 17.71)$ & $38.90( \pm 4.01)$ & 23.706 & $7.11 \times 10^{-6}$ & 0.0136 & 0.82 & $4.15 \times 10^{-5}$ \\
\hline CMA & $4.57( \pm 11.99)$ & $11.06( \pm 9.60)$ & $31.05( \pm 6.50)$ & 27.906 & $8.71 \times 10^{-5}$ & $1.89 \times 10^{-6}$ & $5.11 \times 10^{-4}$ & 0.05 \\
\hline TTA & $35.54( \pm 21.44)$ & $17.51( \pm 22.93)$ & $8.92( \pm 3.78)$ & 11.336 & 0.0034 & 0.038 & 0.80 & 0.056 \\
\hline
\end{tabular}

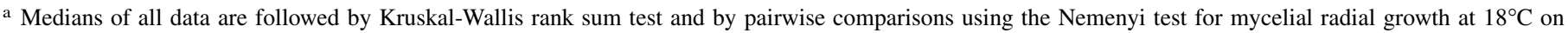
potato dextrose agar (PDA), corn meal agar (CMA), and tree tomato agar (TTA). Significant $P$ values are shown in bold.

TABLE 3. Mycelial radial growth within Phytophthora species collected from central, southern Colombian regions, Ecuador, and the United States ${ }^{\mathrm{a}}$

\begin{tabular}{|c|c|c|c|c|c|c|c|c|c|c|}
\hline \multirow[b]{4}{*}{ Media } & \multirow[b]{4}{*}{ Species } & \multicolumn{9}{|c|}{ Median radial growth $\left(\mathrm{cm}^{2}\right)$} \\
\hline & & \multirow[b]{3}{*}{ Central } & \multirow[b]{3}{*}{ Southern } & \multirow[b]{3}{*}{ U.S.A. } & \multirow[b]{3}{*}{ Ecuador } & \multirow{2}{*}{\multicolumn{2}{|c|}{$\begin{array}{l}\text { Kruskal-Wallis } \\
\text { rank sum test }\end{array}$}} & \multicolumn{3}{|c|}{$\begin{array}{c}\text { Pairwise comparisons using } \\
\text { Nemenyi test }\end{array}$} \\
\hline & & & & & & & & \multirow{2}{*}{$\begin{array}{c}\text { Central } \\
\text { versus } \\
\text { southern }\end{array}$} & \multirow{2}{*}{$\begin{array}{c}\text { Central } \\
\text { versus } \\
\text { U.S.A. }\end{array}$} & \multirow{2}{*}{$\begin{array}{c}\text { Southern } \\
\text { versus } \\
\text { U.S.A. }\end{array}$} \\
\hline & & & & & & $\chi^{2}, \mathrm{df}=2$ & $P$ value & & & \\
\hline \multirow[t]{3}{*}{ PDA } & P. betacei & $27.411 \pm(23.83)$ & $0 \pm(0.51)$ & - & - & 37.24 & $8.20 \times 10^{-9}$ & $1.42 \times 10^{-9}$ & - & - \\
\hline & $P$. infestans & $31.817 \pm(19.14)$ & $32.448 \pm(10.36)$ & $39.086 \pm(9.99)$ & - & 2.37 & 0.305 & 1 & 0.97 & 0.88 \\
\hline & P. andina & - & - & - & $38.899 \pm(4.01)$ & - & - & - & - & - \\
\hline \multirow[t]{3}{*}{ CMA } & P. betacei & $3.506 \pm(2.00)$ & $27.498 \pm(11.48)$ & - & - & 24.28 & $5.34 \times 10^{-6}$ & $8.45 \times 10^{-7}$ & - & - \\
\hline & P. infestans & $9.876 \pm(9.60)$ & $11.626 \pm(10.33)$ & $20.641 \pm(6.22)$ & - & 6.01 & 0.049 & 1 & 0.18 & 1 \\
\hline & $P$. andina & - & - & - & $31.046 \pm(6.50)$ & - & - & - & - & - \\
\hline \multirow[t]{3}{*}{ TTA } & P. betacei & $42.970 \pm(26.63)$ & $34.639 \pm(6.16)$ & - & - & 0.97 & 0.617 & 0.54 & - & - \\
\hline & P. infestans & $22.805 \pm(23.96)$ & $14.492 \pm(8.87)$ & $0.286 \pm(0.61)$ & - & 13.99 & 0.001 & 0.16 & 0.01 & 0.45 \\
\hline & $P$. andina & - & - & - & $8.925 \pm(3.78)$ & - & - & - & - & - \\
\hline
\end{tabular}

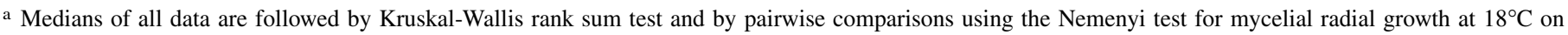
potato dextrose agar (PDA), corn meal agar (CMA), and tree tomato agar (TTA). 
morphological and molecular approaches. Strains of P. infestans were associated with potato crops ( $S$. tuberosum and $S$. phureja), while strains of $P$. betace $i$ were associated with tree tomato crops (S. betaceum), as it was previously described in southern Colombia (Mideros et al. 2018). Also, both species appear clonal based on the linkage disequilibrium analyses. Our results expand the knowledge about the geographic distribution of $P$. betacei, which is now known to span from the central to the southern region of Colombia. In central Colombia, the species $P$. betacei is also restricted to tree tomato and clearly differentiates from $P$. infestans. Furthermore, based on the diversity results and clonality, we hypothesize that $P$. betacei originated in the Southern region of Colombia and only some genotypes have migrated to the center. Very few studies have been performed on tree tomato in Colombia to support the hypothesis of this southern origin, but the culture of this crop is more common in the south and the exchange of seeds is limited among regions. These facts could contribute to maintain a higher level of diversity of the pathogen in the south.

$P$. betacei infects tree tomato crops in the central region (Cundinamarca) of Colombia. The genetic analyses from the

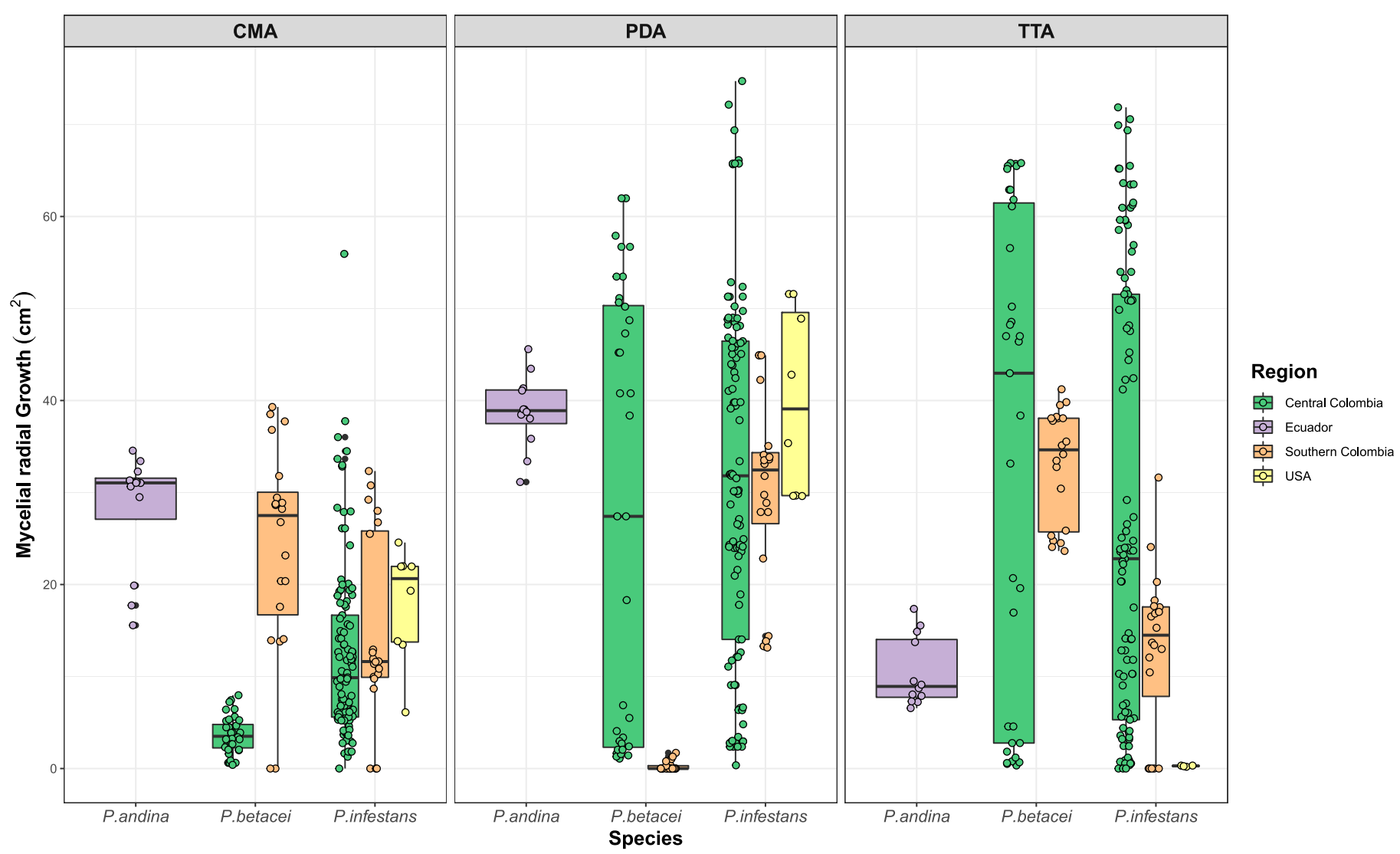

Fig. 3. Mycelial radial growth among Phytophthora species from four geographical regions. Effect of species and region on mycelial radial growth on potato dextrose agar (PDA), corn meal agar (CMA), and tree tomato agar (TTA) 15 days after incubation.

TABLE 4. Comparisons of all quantitative morphological traits (length, width, length/width, and area) of sporangia among Phytophthora betacei, P. infestans, and P. andina $^{\text {a }}$

\begin{tabular}{|c|c|c|c|c|c|c|c|c|c|}
\hline \multirow[b]{3}{*}{ Traits } & \multirow[b]{3}{*}{ Medium } & & & & \multirow{2}{*}{\multicolumn{2}{|c|}{$\begin{array}{l}\text { Kruskal-Wallis } \\
\text { rank sum test }\end{array}$}} & \multicolumn{3}{|c|}{ Pairwise comparisons using Nemenyi test } \\
\hline & & \multicolumn{3}{|c|}{ Median } & & & \multirow{2}{*}{$\begin{array}{l}\text { P. betacei } \\
\text { versus } \\
P . \text { andina }\end{array}$} & \multirow{2}{*}{$\begin{array}{l}\text { P. infestans } \\
\text { versus } \\
\text { P. andina }\end{array}$} & \multirow{2}{*}{$\begin{array}{l}\text { P. infestans } \\
\text { versus } \\
\text { P. betacei }\end{array}$} \\
\hline & & P. betacei & P. infestans & P. andina & & $P$ value & & & \\
\hline \multirow{2}{*}{$\begin{array}{l}\text { sporangia } \\
(\mu \mathrm{m})\end{array}$} & CMA & $36.31 \pm(5.79)$ & $1.86 \pm(6.21)$ & 37. & 61.03 & $5.6 \times 10^{-14}$ & 1 & $1.93 \times 10^{-5}$ & $3.16 \times 10^{-12}$ \\
\hline & TTA & $37.06 \pm(6.81)$ & $32.77 \pm(5.39)$ & $37.43 \pm(4.99)$ & 86.66 & $2.2 \times 10^{-16}$ & 1 & $1.69 \times 10^{-10}$ & $1.29 \times 10^{-13}$ \\
\hline $\begin{array}{l}\text { Width of } \\
\text { sporangia } \\
(\mu \mathrm{m})\end{array}$ & PDA & 16.44 & 19.3 & $18.63 \pm($ & 58.04 & $2.5 \times 10^{-13}$ & $1.44 \times 10^{-4}$ & 00 & $9.78 \times 10^{-13}$ \\
\hline \multirow{3}{*}{$\begin{array}{l}\text { Length/ } \\
\text { width } \\
\text { ratio }(\mu \mathrm{m})\end{array}$} & PDA & $1.94 \pm(0.68)$ & $1.61 \pm(0.27)$ & $1.97 \pm(0.05)$ & 156.23 & $2.2 \times 10^{-16}$ & 0.1 & 0 & $1.29 \times$ \\
\hline & CMA & $2.20 \pm(0.26)$ & $1.67 \pm(0.29)$ & $2.06 \pm(0.02)$ & 281.45 & $2.2 \times 10^{-16}$ & $5.64 \times 10^{-6}$ & $1.57 \times 10^{-8}$ & $\mathbf{0}$ \\
\hline & TTA & $2.46 \pm(1.47)$ & $1.62 \pm(0.29)$ & $2.16 \pm(0.18)$ & 351.34 & $2.2 \times 10^{-16}$ & $8.17 \times 10^{-6}$ & $1.57 \times 10^{-13}$ & $\mathbf{0}$ \\
\hline \multirow{2}{*}{$\begin{array}{l}\text { Mean of } \\
\text { sporangia } \\
\text { area }\left(\mu \mathrm{m}^{2}\right)\end{array}$} & $\mathrm{PD}$ & 1,575 . & $1,902.27 \pm$ & $2,179.61 \pm$ & 25.43 & $3.0 \times 10^{-6}$ & & 0.02 & $2.34 \times 10^{-3}$ \\
\hline & CMA & $1,846.83 \pm(542.02)$ & $1,922.96 \pm(596.21)$ & $2,110.48 \pm(464.76)$ & 2.09 & 0.3517 & 0.98 & 1 & 1 \\
\hline
\end{tabular}

${ }^{a}$ Strains growing at $18^{\circ} \mathrm{C}$ on potato dextrose agar (PDA), corn meal agar (CMA), and tree tomato agar (TTA). Medians of all data are followed by the KruskalWallis rank sum test results and a pairwise comparisons using Nemenyi test. Statistically significant $P$ values are shown in bold. 
PCA based on 12 SSR loci showed a clear distinction between Phytophthora spp. strains collected from potato and tree tomato crops. Phytophthora spp. strains collected on tree tomato crops were associated with a new species, $P$. betacei, whereas Phytophthora spp. strains collected on potato crops were associated with $P$. infestans. $P$. betace $i$ was previously reported infecting tree tomato crops in the southern region of Colombia and was classified according to morphological, physiological, molecular, and host preference approaches (Mideros et al. 2018).

The line of evidence for the distinction between $P$. betace $i$ strains and $P$. infestans was the genetic differentiation provided by the SSR data. Here, we evaluated a set of 12 SSR, six of them used by Mideros et al. (2018) and Cooke et al. (2012), and we included a set of additional microsatellite loci reported by Li et al. (2013) to detect the genetic differentiation between both species $(P$. betacei and $P$. infestans). Our results highly support the distinction between these species. All strains collected from tree tomato crops in the central region grouped with $P$. betacei strains recovered from the

TABLE 5. Coefficients of the linear discriminant (LD) analysis for the quantitative morphological traits of Phytophthora strains ${ }^{\mathrm{a}}$

\begin{tabular}{lcc}
\hline Character & LD1 & LD2 \\
\hline Length of sporangia $(\mu \mathrm{m})$ & 0.067 & 0.700 \\
Width of sporangia $(\mu \mathrm{m})$ & -0.104 & -1.075 \\
Length/width ratio & $\mathbf{1 . 4 6 1}$ & $\mathbf{- 1 1 . 5 2}$ \\
Area of sporangia $(\mu \mathrm{m})$ & 0.003 & -0.002 \\
\hline
\end{tabular}

a Values in bold highlight the variables that bear the most information about the distribution of the data on each linear discriminant function (LD1 and LD2) and allow to differentiate $P$. betacei from $P$. infestans and $P$. andina. southern region of Colombia (Mideros et al. 2018) and were completely separated from $P$. infestans strains collected from potato fields. These results confirm the genetic differentiation of $P$. betace $i$ and suggest that $P$. betacei is the only Phytophthora species infecting tree tomato crops in the country.

Our PCA analyses that included $P$. andina strains collected from S. quitoense and Anarrhichomenum complex crops, of the EC-2 clonal lineage, grouped Phytophthora strains into at least three major genetic groups with distinct host preferences: (i) P. betacei; (ii) P. infestans (collected from potato and tomato crops); and (iii) $P$. andina [collected from naranjilla (S. quitoense] and Anarrhichomenum complex hosts). These results confirm the monophyletic status of $P$. betacei, which is isolated from the other sister species in the 1c clade ( $P$. andina and $P$. infestans).

In previous studies, in addition to genetic variation, the second line of evidence to distinguish $P$. betace $i$ was supported by the morphological analyses based on seven morphological traits including: length, width, length/width ratio, area of sporangia, hyphal width, and mycelial radial growth on different media (Mideros et al. 2018). Here we evaluated four of these morphological traits (length of sporangia, width of sporangia, area of sporangia, and the length/width ratio of sporangia on three different media) to support the morphological differentiation between $P$. betacei and P. infestans. Similar to Mideros et al. (2018), the discriminant analysis showed that the length/width ratio of sporangia was the most determinant trait in differentiating among the Phytophthora strains. The evident differences in morphological traits did support the hypothesis that the two groups of strains represent two genetically distinct species supported by the molecular approach. Furthermore, $P$. andina strains are hardly differentiated from the two other species, suggesting that no

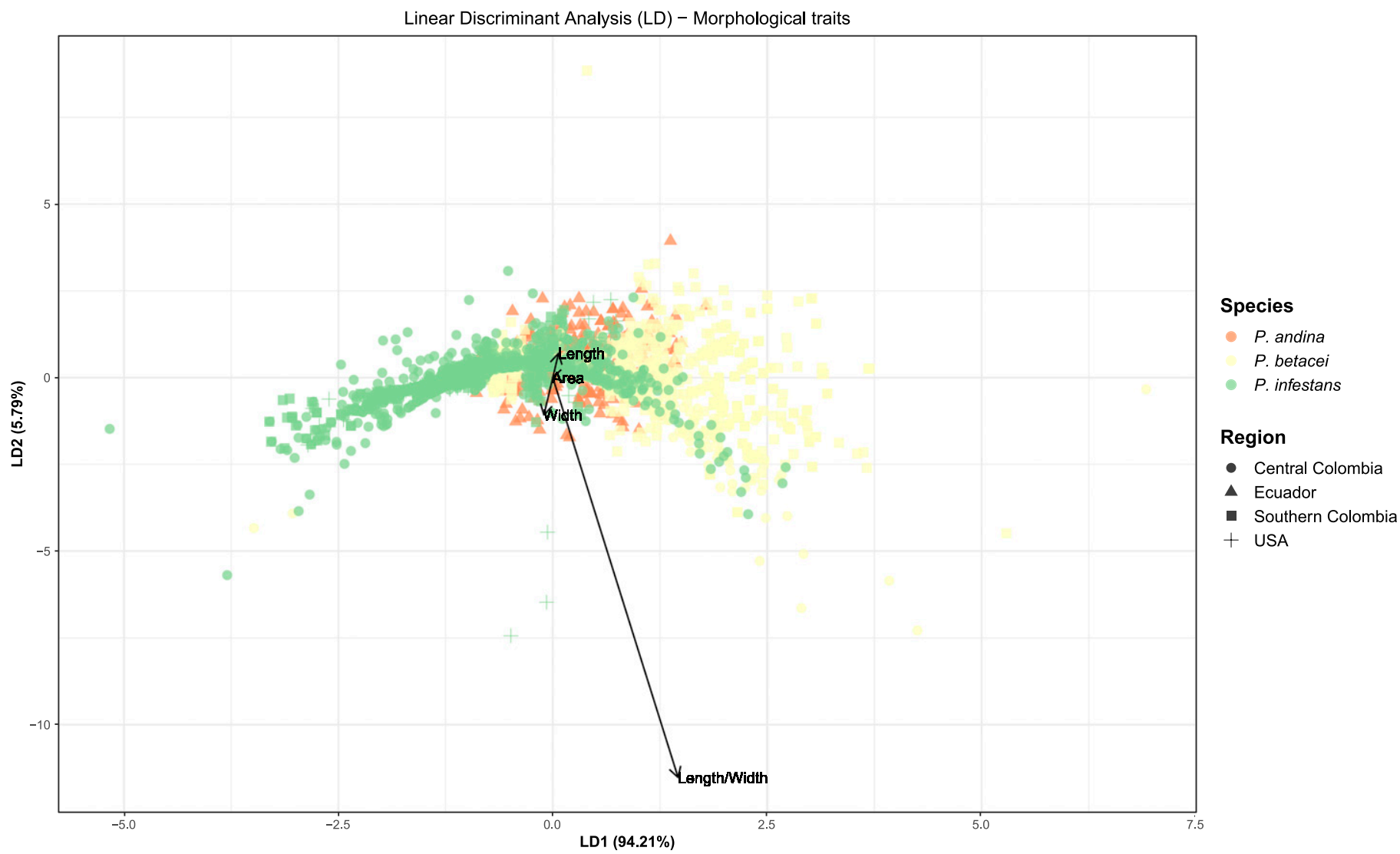

Fig. 4. Plot of the morphological traits of Phytophthora species from four geographical regions using a discriminant analysis (DA) showing the first and second discriminant components. P. betacei strains are shown in light gray/yellow, $P$. infestans in dark gray/green, and $P$. andina in medium gray/orange. Strains collected from the central region are represented with circles, from the southern region with squares, from Ecuador with triangles, and from the United States with crosses. The arrows represent the magnitude and direction of the coefficients of the linear discriminant (LD) components. 
significant variation in morphology could be detected because of potentially high phenotypic plasticity in all Phytophthora strains (Pais et al. 2018).

Population genetic structure of $P$. infestans and $P$. betacei is also defined by geographical origin. We also compared $P$. infestans strains collected from cultivated potato and $P$. betace $i$ strains collected from tree tomato in the central region, with strains from the same hosts collected from the southern region of Colombia (strains from this study and Mideros et al. [2018]). Our results suggested a weak but consistent genetic population structure of each species, $P$. infestans and $P$. betacei, according to the geographic origin of the strains. P. infestans strains appear spatially structured with two main groups. These groups correspond to strains collected from the central region and strains collected from the southern region of Colombia. A similar pattern was found for $P$. betace $i$ where two genetic groups were detected associated with the geographic origin (central and southern of Colombia). These data suggest a correlation between genetic diversity and geographic origin of the strains independent of the host species. The minimum-spanning network showed that for both species almost no haplotypes were shared between southern and central strains.

We also detected a variation in the genetic diversity according to the geographic origin. Strains collected on potato crops in the central region of the country were genetically more diverse than strains collected on potato crops in the south (with some but not all the indexes calculated). Similar results were found for $P$. betacei strains despite the low genetic diversity reported in this species. $P$. betace $i$ strains collected in the southern region were more diverse than $P$. betace $i$ strains collected in the central region.

In conclusion, this study confirms that $P$. infestans is the predominant species causing late blight disease on cultivated potatoes and $P$. betace $i$ is the predominant species attacking tree tomato crops in Colombia. Molecular data support the genetic differentiation of $P$. betace $i$ and its closely related species from the Phytophthora 1c clade (P. infestans and P. andina) and confirm the taxonomic status of this nascent species originally reported in the south of Colombia. Furthermore, our results show the extended geographic distribution of $P$. betacei, spanning from central to southern Colombia (Mideros et al. 2018).

We also suggest that the population genetic structure of Phytophthora can be shaped by the combined effect of the genetic variation across species ( $P$. infestans and $P$. betacei) and the geographic origin of the strain (central or southern region). Although the effect of the geographic origin was low, different genotypes can be associated with the central or southern region for each species. Further studies are needed to test in detail if host specificity of $P$. betace $i$ strains in the central region is the main ecological mechanism keeping $P$. betace $i$ in reproductive isolation from $P$. infestans strains.

\section{LITERATURE CITED}

Adler, N. E., Erselius, L. J., Chacon, M. G., Flier, W. G., and Ordonez, M. E. 2004. Genetic diversity of Phytophthora infestans sensu lato in Ecuador provides new insight into the origin of this important plant pathogen. Phytopathology 94:154-162.

Agapow, P. M., and Burt, A. 2001. Indices of multilocus linkage disequilibrium. Mol. Ecol. Notes 1:101-102.

Bates, D., Maechler, M., Bolker, B., and Walker, S. 2014. Ime4: Linear MixedEffects Models Using Eigen and S4. R package Version 1.

Bruvo, R., Michiels, N. K., D'Souza, T. G., and Schulenburg, H. 2004. A simple method for the calculation of microsatellite genotype distances irrespective of ploidy level. Mol. Ecol. 13:2101-2106.

Cárdenas, M., Grajales, A., Sierra, R., Rojas, A., González-Almario, A., Vargas, A., Marín, M., Fermín, G., Lagos, L. E., and Grünwald, N. J. 2011. Genetic diversity of Phytophthora infestans in the Northern Andean region. BMC Genet. 12:23.

Cavalier-Smith, T. 1993. Kingdom Protozoa and its 18 phyla. Microbiol. Rev. 57:953-994
Chao, A., and Shen, T. J. 2003. Nonparametric estimation of Shannon's index of diversity when there are unseen species in sample. Environ. Ecol. Stat. 10:429-443.

Cooke, D. E. L., Cano, L. M., Raffaele, S., Bain, R. A., Cooke, L. R., Etherington, G. J., Deahl, K. L., Farrer, R. A., Gilroy, E. M., Goss, E. M., Grünwald, N. J., Hein, I., MacLean, D., McNicol, J. W., Randall, E., Oliva, R. F., Pel, M. A., Shaw, D. S., Squires, J. N., Taylor, M. C., Vleeshouwers, V. G. A. A., Birch, P. R. J., Lees, A. K., and Kamoun, S. 2012. Genome analyses of an aggressive and invasive lineage of the Irish potato famine pathogen. PLoS Pathog 8:e1002940.

Correll, D. S. 1962. The potato and its wild relatives. Contrib. Tex. Res. Found. Bot. Stud. 4:1-606.

Csardi, G., and Nepusz, T. 2006. The igraph software package for complex network research. InterJournal, Complex Systems 1695. https:// igraph.org

Danies, G., Myers, K., Mideros, M., Restrepo, S., Martin, F., Cooke, D., Smart, C., Ristaino, J., Seaman, J., Gugino, B., Grünwald, N., and Fry, W. E. 2014. An ephemeral sexual population of Phytophthora infestans in the Northeastern United States and Canada. PLoS One 12:1-21.

Danies, G., Small, I. M., Myers, K., Childers, R., and Fry, W. E. 2013. Phenotypic characterization of recent clonal lineages of Phytophthora infestans in the United States. Plant Dis. 97:873-881.

Dick, M. W. 1995. Sexual reproduction in the Peronosporomycetes (chromistan fungi). Can. J. Bot. 73:712-724.

Forbes, G. A., Escobar, X. C., Ayala, C. C., Revelo, J., Ordoñez, M. E., Fry, B. A., Doucett, K., and Fry, W. E. 1997. Population genetic structure of Phytophthora infestans in Ecuador. Phytopathology 87:375-380.

Fry, W. E. 2008. Phytophthora infestans: The plant (and R gene) destroyer. Mol. Plant Pathol. 9:385-402.

Gómez-Alpizar, L., Hu, C.-H., Oliva, R., Forbes, G., and Ristaino, J. B. 2008. Phylogenetic relationships of Phytophthora andina, a new species from the highlands of Ecuador that is closely related to the Irish potato famine pathogen Phytophthora infestans. Mycologia 100:590-602.

Goodwin, S. B., Spielman, L. J., Matuszak, J. M., Bergeron, S. N., and Fry, W. E. 1992. Clonal diversity and genetic differentiation of Phytophthora infestans populations in northern and central Mexico. Phytopathology 82: 955-961.

Goss, E. M., Cardenas, M. E., Myers, K., Forbes, G. A., Fry, W. E., Restrepo, S., and Grünwald, N. J. 2011. The plant pathogen Phytophthora andina emerged via hybridization of an unknown Phytophthora species and the Irish potato famine pathogen, $P$. infestans. PLoS One 6:e24543.

Goss, E. M., Tabima, J., Cooke, D., Restrepo, S., Fry, W. E., Forbes, G., Fieland, V., Cárdenas, M., and Grünwald, N. J. 2014. The Irish potato famine pathogen Phytophthora infestans originated in central Mexico rather than the Andes. Proc. Nat. Acad. Sci. 111:8791-8796.

Griffith, G. W., and Shaw, D. S. 1998. Polymorphisms in Phytophthora infestans: Four mitochondrial haplotypes are detected after PCR amplification of DNA from pure cultures or from host lesions. Appl. Environ. Microbiol. 64:4007-4014.

Grünwald, N. J., and Hoheisel, G.-A. 2006. Hierarchical analysis of diversity, selfing, and genetic differentiation in populations of the oomycete Aphanomyces euteiches. Phytopathology 96:1134-1141.

Jombart, T. 2008. Adegenet: A R package for the multivariate analysis of genetic markers. Bioinformatics 24:1403-1405.

Kamvar, Z. N., Tabima, J. F., and Grünwald, N. J. 2014. Poppr: An R package for genetic analysis of populations with clonal, partially clonal, and/or sexual reproduction. PeerJ 2:e281.

Knapova, G., and Gisi, U. 2002. Phenotypic and genotypic structure of Phytophthora infestans populations on potato and tomato in France and Switzerland. Plant Pathol. 51:641-653.

Kroon, L. P. N. M., Bakker, F. T., van den Bosch, G. B. M., Bonants, P. J. M., and Flier, W. G. 2004. Phylogenetic analysis of Phytophthora species based on mitochondrial and nuclear DNA sequences. Fungal Genet. Biol. 41: 766-782.

Kumar, J., Nelsen, R. J., and Zeigler, R. S. 1999. Population structure and dynamics of Magnaporthe grisea in the Indian Himalayas. Genetics 152: 971-984.

Lees, A. K., Wattier, R., Shaw, D. S., Sullivan, L., Williams, N. A., and Cooke, D. E. L. 2006. Novel microsatellite markers for the analysis of Phytophthora infestans populations. Plant Pathol. 55:311-319.

Li, Y., Cooke, D. E., Jacobsen, E., and Van Der Lee, T. 2013. Efficient multiplex simple sequence repeat genotyping of the oomycete plant pathogen Phytophthora infestans. J. Microbiol. Methods 92:316-322.

Martin, F. N., Tooley, P. W., and Blomquist, C. 2004. Molecular detection of Phytophthora ramorum, the causal agent of sudden oak death in California, and two additional species commonly recovered from diseased plant material. Phytopathology 94:621-631.

Mayton, H., Smart, C. D., Moravec, B. C., Mizubuti, E. S. G., Muldoon, A. E., and Fry, W. E. 2000. Oospore survival and pathogenicity of single oospore 
recombinant progeny from a cross involving US-17 and US-8 genotypes of Phytophthora infestans. Plant Dis. 84:1190-1196.

Mideros, M. F., Turissini, D. A., Guayazán, N., Ibarra-Avila, H., Danies, G., Cárdenas, M., Myers, K., Tabima, J., Goss, E. M., Bernal, A., Lagos, L. E., Grajales, A., Gonzalez, L. N., Cooke, D. E. L., Fry, W. E., Grünwald, N., Matute, D. R., and Restrepo, S. 2018. Phytophthora betacei, a new species within Phytophthora clade 1c causing late blight on Solanum betaceum in Colombia. Persoonia 41:39-55.

Montarry, J., Corbiere, R., and Andrivon, D. 2007. Is there a trade-off between aggressiveness and overwinter survival in Phytophthora infestans? Funct. Ecol. 21:603-610.

Nei, M. 1987. Molecular Evolutionary Genetics. Columbia University Press, New York.

Oliva, R. F., Flier, W. G., Ristaino, J. B., and Forbes, G. A. 2010. Phytophthora andina sp. Nov., a newly identified heterothallic pathogen of solanaceous hosts in the Andean highlands. Plant Pathol. 59:613-625.

Ordoñez, M. E., Hohl, H. R., Velasco, A., Ramon, M. P., Oyarzun, P. J., Smart, C. D., Fry, W. E., Forbes, G. A., and Erselius, L. J. 2000. A novel population of Phytophthora, similar to P. infestans, attacks wild Solanum species in Ecuador. Phytopathology 90:197-202.

Oyarzún, P. J., Pozo, A., Ordoñez, M. E., Doucett, K., and Forbes, G. A. 1998. Host specificity of Phytophthora infestans on tomato and potato in Ecuador. Phytopathology 88:265-271.

Pais, M., Yoshida, K., Giannakopoulou, A., Pel, M. A., Cano, L. M., Oliva, R. F., Witek, K., Lindqvist-Kreuze, H., Vleeshouwers, V. G. A. A., Kamoun, S. 2018. Gene expression polymorphism underpins evasion of host immunity in an asexual lineage of the Irish potato famine pathogen. BMC Evol. Biol. 18:93.

Pohlert, T. 2014. The Pairwise Multiple Comparison of Mean Ranks Package (PMCMR). R Package. http://CRAN.R-project.org/package=PMCMR
R Core Team. 2013. R: A Language and Environment for Statistical Computing. R Foundation for Statistical Computing, Vienna, Austria. http:// www.R-project.org/

Rueden, C. T., Schindelin, J., Hiner, M. C., DeZonia, B. E., Walter, A. E., Arena, E. T., and Eliceiri, K. W. 2017. ImageJ2: ImageJ for the next generation of scientific image data. BMC Bioinform. 18:529.

Santana, F. M., and Gomes, C. B. 2013. Characterization of Phytophthora infestans populations of southern Brazil in 2004 and 2005. Phytoparasitica 41:557-568.

Schindelin, J., Rueden, C. T., Hiner, M. C., and Eliceiri, K. W. 2015. The ImageJ ecosystem: An open platform for biomedical image analysis. Mol. Reprod. Dev. 82:518-529.

Shannon, C. E. 2001. A mathematical theory of communication. ACM Sigmobile Mobile Computing and Communications Review 5:3-55.

Silva, B., Jaramillo, S., and Marín, M. 2009. Caracterización genética de aislamientos de Phytophthora infestans en las zonas productoras de papa de los departamentos de Antioquia, Boyacá, Cundinamarca y Norte de Santander (Colombia). Actual. Biol. 31:5-20.

Stoddart, J. A., and Taylor, J. F. 1988. Genotypic diversity: Estimation and prediction in samples. Genetics 118:705-711.

Vargas, A. M., Quesada, L. M., Céspedes, M. C., Carreño, N., González, A., Rojas, A., Paola, A. Z., Myers, K., Fry, W. E., Jiménez, P., Bernal, A. J., and Restrepo, S. 2009. Characterization of Phytophthora infestans populations in Colombia: First report of the A2 mating type. Phytopathology 99:82-88.

Venables, W. N., and Ripley, B. D. 2002. Modern Applied Statistics with S. Issues of Accuracy and Scale (Fourth). Springer, New York.

Yoon, H. S., Hackett, J., Pinto, G., and Bhattacharya, D. 2002. The single, ancient origin of chromist plastids. Proc. Natl. Acad. Sci. USA 99:15507-15512. 